quatro variedades. A maioria das espécies $(60 \%)$ foi encontrada em baixio. Analisou-se a distribuição das espécies ao longo das parcelas, verificando-se as suas preferências aos tipos de solos e habitats. Estudou-se também a similaridade da pteridoflora entre as diferentes posições topográficas da área de estudo. Foram efetuadas comparações com a ocorrência de pteridófitas em uma área de 0,5 hectare onde constatou-se que 10 espécies foram comuns em ambas as reservas. Em torno de $68 \%$ das espécies do Campus são terrestres, $24 \%$ são epifitas, $8 \%$ tiveram ambas preferências. O menor número de espécies encontrado no Campus comparado com o da Reserva Ducke sugere perturbações antrópicas nessas florestas. Com as pteridófitas do Campus elaborou-se uma chave geral de familias, gêneros, espécies e variedades que fornecerá subsidios a futuros taxônomos deste grupo de plantas.

Palavras-chave: Pteridófita, Amazônia, composição floristica, similaridade Agência Financiadora: CNPq, INPA, UA

TíTULO: Estabelecimento de plântulas de Copaifera multijuga Hayne (Caesalpiniaceae) em fragmentos florestais e estádios de sucessão

AUTORA: Maria Elisabeth de Assis Elias

DATA: 11/abril/1997

LOCAL: Instituto Nacional de Pesquisas da Amazônia; Coordenação de Pesquisas em Botânica, Manaus-AM

NÍVEL: Mestrado

BANCA EXAMINADORA: Isolde Dorothea Kossmann Ferraz - INPA (orientadora)

Rita de Cassia Mesquita - SMITHSONIAN-INPA

Gil Vieira - INPA

RESUMO - Neste bioensaio foi comparado o estabelecimento de plântulas de Copaifera multijuga Hayne em função de diferentes niveis de perturbação florestal, com as igualmente transplantadas na floresta primária. C. multijuga mostrou maior sobrevivência (80-95\%) na floresta primária e nos fragmentos florestais de 100 e 10 hectares. Nestas condições sombreadas as plântulas são suprimidas sem ou com pouco crescimento. A menor sobrevivência foi observada na pastagem, porém neste habitat, as plântulas apresentaram maior biomassa seca final $(4,0 \mathrm{~g})$, sendo que a floresta secundária foi o ambiente com melhor performance: alta sobrevivência $(80 \%)$ e bom crescimento $(1,9 \mathrm{~g})$. Os resultados mostraram que a espécie C. multijuga na sua fase inicial é tolerante ao sombreamento, podendo sobreviver durante muito tempo em estado suprimido, porém necessita de luminosidade para o seu desenvolvimento.

Palavras-chave: Copaifera multijuga, sucessão florestal, fragmentos florestais, plântulas, estabelecimento

Agência financiadora: CAPES, SMITHSONIAN

\title{
Museu Nacional - UFRJ
}

TíTULO: As espécies brasileiras do gênero Trigonia Aubl. (Trigoniaceae) na região sudeste

AUTOR: João Rodrigues Miguel

DATA: $\quad 10 /$ janeiro/1996

LOCAL: Universidade Federal do Rio de Janeiro, Museu Nacional, Departamento de Botânica, Rio de Janeiro, RJ

NÍVEL: Mestrado

BANCA EXAMINADORA: Jorge Fontella Pereira - JBRJ (orientador)

Luiz Emygdio de Mello Filho - UFRJ

Graziela Maciel Barroso - JBRJ

Jorge Pedro Carauta - FEEMA 
RESUMO-A familia Trigoniaceae End. está representada na região sudeste brasileira por dois gêneros: um monotipico, Trigoniodendrom E. F. Guim. \& Miguel, e o outro com dezesseis espécies, Trigonia Aubl. Este trabalho trata da revisão taxonômica do gênero Trigonia na região. Para tanto além da pesquisa bibliográfica, foram feitas diversas excursões para observações de campo, coleta de material e fotografias. Este trabalho conta com descrições para a familia, gênero, espécies e variedades do grupo, acrescido de chaves analiticas e ilustrações.

Palavras-chave: taxonomia, Trigoniaceae, Trigonia

Agência financiadora: $\mathrm{CNPq}$

TíTULO: A família Bromeliaceae na Reserva Ecológica Rio das Pedras, Mangaratiba, Rio de Janeiro, Brasil

AUTOR: Ula de Andrade Vidal

DATA: $\quad 29 / \operatorname{março} / 1996$

LOCAL: Universidade Federal do Rio de Janeiro, Museu Nacional, Departamento de Botânica, Rio de Janeiro, RJ

NIVEL: Mestrado

BANCA EXAMINADORA: Graziela Maciel Barroso - JBRJ (orientador)

Luiz Emygdio de Mello Filho - UFRJ

Regina Helena P. Andreata - USU

Gustavo Martinelli - JBRJ

RESUMO-O trabalho refere-se à flora da familia Bromeliaceae da Reserva Ecológica Rio das Pedras, localizada em Mangaratiba, Estado do Rio de Janeiro, Brasil. Foram identificadas 19 espécies da familia Bromeliaceae, com representantes subordinados às três subfamilias: Pitcairnioideae com uma espécie; Tillandsioideae, com sete espécies; e Bromelioideae, com 11 espécies. O trabalho teve como objetivo contribuir para o conhecimento da diversidade floristica das Bromeliaceae na Mata Atlântica da região sudeste do Brasil e facilitar a identificação das espécies estudadas, através de descrições morfológicas, estampas, mapas de distribuição geográfica e comentários sobre o hábito das espécies em seu ambiente natural.

Palavras-chave: Bromeliaceae, Mata Atlântica, flora Agência financiadora: $\mathrm{CNPq}$

TÍTULO: Aspectos morfológicos do gênero Ceramium Roth (Ceramiaceae, Rhodophyta) no Estado do Rio de Janeiro.

AUTOR: Maria Beatriz B. de B. Barreto

DATA: $\quad 08 / \mathrm{maio} / 1996$

LOCAL: Universidade Federal do Rio de Janeiro, Museu Nacional, Departamento de Botânica, Rio de Janeiro, RJ

NÍVEL: Mestrado

BANCA EXAMINADORA: Yocie Yoneshigue -Valentin - UFRJ (orientador)

Mariângela Menezes - UFRJ

Márcia Abreu de O. Figueiredo - JBRJ

Sílvia Maria P. de B. Guimarães - IBt

RESUMO -Os caracteres taxonômicos mais importantes na identificação das espécies do gênero Ceramium, foram o padrão de desenvolvimento do nó a partir das células periaxiais e o padrão de ramificação. De acordo com esse diagnóstico, foram identificadas 11 espécies no Estado do Rio de Janeiro: C. brasiliense, C. brevizonatum var. caraibicum, C. comptum, C. flaccidum, C. leutzelburgii, C. strictum, C. tenerrimum, C. temuissimum, C. vagabunde e Ceramium sp., pertencendo ao subgênero Hormoceras, pela caracterização do talo em nó coticado e entre-nó ecorticado e C. atlanticum, 
representando o subgênero euceramium com entrenó coberto por células basípetas oriundas do córtex nodal. C. flaccidum e C. tenerrimum apresentaram variação morfológica com C. dawsoni e C. brevizonatum var. caraibicum respectivamente. Portanto, faz-se necessária a observação do material tipo para definir a posição taxonômica destes táxons.

Palavras-chave: Ceramium, taxonomia de Rodófita, algas marinhas Agência financiadora: $\mathrm{CNPq}$

TÍTULO: Diospyros Dalech. ex L. (Ebenaceae) do Estado do Rio de Janeiro

AUTOR: Rosana Conrado Lopes

DATA: $\quad 29 / \mathrm{maio} / 1996$

LOCAL: Universidade Federal do Rio de Janeiro, Museu Nacional, Departamento de Botânica, Rio de Janeiro, RJ

NÍVEL: Mestrado

BANCA EXAMINADORA: Graziela Maciel Barroso - JBRJ (orientador)

Maria Célia B. Pinheiro - UFRJ

Lúcia D'Ávila F. de Carvalho - JBRJ

Regina Helena P. Andreata - USU

RESUMO-O presente trabalho trata do estudo taxonômico das espécies de Diospyros Dalech. ex L., pertencente a família Ebenaceae, ocorrentes no Estado do Rio de Janeiro. Estas espécies são aqui redescritas, ilustradas e ordenadas em chave analitica que facilita sua identificação. É feito um estudo comparativo dos padrões de venação das folhas, dos tipos de inflorescências e da morfologia dos frutos. São representadas as épocas de floração e frutificação, além de informações referentes ao habitat, nome vulgar e utilidade de cada espécie, bem como uma lista dos nomes dos coletores e mapas de distribuição geográfica.

Palavras-chave: Ebenaceae, Diospyros

Agência financiadora: CAPES

TíTULO: Palinotaxonomia de espécies das tribos Gardenieae A. Rich. ex. DC. e Spermacoceae A. Rich. ex. Dum. (Rubiaceae Juss.) ocorrentes nas restingas do Estado do Rio de Janeiro

AUTOR: Sonia Regina de Melo Crespo

DATA: $\quad 12 /$ julho/ 1996

LOCAL: Universidade Federal do Rio de Janeiro, Museu Nacional, Departamento de Botânica, Rio de Janeiro, RJ

NIVEL: Mestrado

BANCA EXAMINADORA: Vania Gonçalves L. Esteves - UFRJ (orientador)

Léa de Jesus Neves - UFRJ

Sigrid Jung Mendaçoli - UNICAMP

Maria Amélia V. da Cruz - IBt

RESUMO- Estudou-se palinologicamente 24 táxons de Rubiaceae Juss. pertencentes às tribos Gardenieae A. Rich. ex DC., subtribo Gardeniiae (Amaioa intermedia var brasiliana (A. Rich) Steyerm., Genipa americana L., Melanopsidium nigrum Cels, Posoqueria latifolia (Rudge) Roem. \& Schult., Randia armata (Sw.) DC., Tocoyena bullata (Vell.) Mart. e Spermacoceae A. Rich. ex Dum. (Borreria brachystemonoides Cham. \& Schlecht., B. capitata (Ruiz \& Pav.) DC., B. cymosa (Spreng.) Cham. \& Schlecht., B. latifolia (Aubl.) K. Schum., B. scabiosoides Cham \& Schlecht., B. verticillata (L.) G. Mey, Diodia apiculata (Wild. ex R. S.) K. Schum, D. gymnocephala (DC.) k. Schum, D. radula (Willd. \& Hoffg ex Roem. \& Schult.) Cham. \& Schlecht., D. teres Walt., Emmeorhiza umbellata (Spreng) K. Schum., Mitracarpus eichleri Schum., M. frigidus (Willd. ex Roem. \& Schult.) K. Schum., M. hirtus (L.) DC., M. Ihotzkiamus Cham., M. megapotamicus (Spreng.) Standl., M. villosus (DC.) Cham. \& Schlecht., Perama hirsuta (Aubl.) ocorrentes nas restingas do Rio de Janeiro. 
Palavras-chave: palinotaxonomia, Rubiaceae, restinga

Agência financiadora: CAPES

TÍTULO: Influência do ciclo hidrológico sobre as variações nictemerais do fitoplâncton de um lago amazônico (Lago Batata, Pará, Brasil)

AUTOR: Sérgio de Melo

DATA: $\quad 12 /$ julho/1996

LOCAL: Universidade Federal do Rio de Janeiro, Museu Nacional, Departamento de Botânica, Rio de Janeiro, RJ

NÍVEL: Mestrado

BANCA EXAMINADORA: Vera Lúcia de Moraes Huszar - UFRJ (orientador)

Francisco de Assis Esteves - UFRJ

Sandra Maria F. O. Azevedo- UFRJ

Fábio Roland - UFJF

RESUMO - O lago Batata $\left(56^{\circ} 1^{\prime} 4^{\prime \prime} \mathrm{e} 56^{\circ} \mathrm{W} 1^{\circ} 28^{\prime} \mathrm{e} 1^{\circ} 33^{\prime} \mathrm{S}\right)$ localiza-se às margens do rio Trombetas, cujo ciclo hidrológico influencia diretamente sua dinâmica. Este estudo teve como objetivo avaliar padrões nictemerais do fitoplânctom do Lago Batata, relacionados ao ciclo hidrológico, e comparar os padrões nictemerais do fitoplânction do lago Batata com outro lago da mesma planície de inundação (Lago Mussurá) no periodo de águas altas. Baseou-se em amostragens nictemerais, realizadas em quatro fases do ciclo hidrológico de setembro de 1989 a junho de 1990 (vazante, águas baixas, enchente e águas altas) mp:agp Batata e na fase de águas altas de 1991 nos Lagos Batata e Mussurá. Distribuição estratificada das algas planctônicas ao longo do ciclo nictemeral foi evidenciada nos periodos em que os lagos apresentaram profundidades superiores a $5 \mathrm{~m}$, exceto nas águas altas de 1991no Lago Batata, quando ocorreram condições de isotermia no periodo noturno. Essa caracteristica diferenciou o Lago Batata do Lago Mussurá. Este último apresentou padrão de distribuição vertical do fitoplâncton estratificado e também maiores densidades.

Palavras-chave: fitotoplâncton, variações nictemerais, lago amazônico Agência financiadora: $\mathrm{CNPq}$

TíTULO: Estudo anatômico de Emilia sonchifolia (L.) DC., Asteraceae, ocorrente em habitats distintos

AUTOR: Vera de Fátima Gomes Alves

DATA: 12/dezembro/1996

LOCAL: Universidade Federal do Rio de Janeiro, Museu Nacional, Departamento de Botânica, Rio de Janeiro, RJ

NIVEL: Mestrado

BANCA EXAMINADORA: Léa de Jesus Neves - UFRJ (orientador)

Cecilia Gonçalves Costa - JBRJ

Maria da Conceição Valente - JBRJ

Maria de Fátima A. Sá - UFRJ

RESUMO-Emilia sonchifolia (L.) DC., espécie popularmente conhecida como serralhinha, pertence a familia Asteraceae, sendo considerada planta daninha, invasora de culturas. Pode ser cultivada como ornamental e ser empregada na medicina caseira, assim como na alimentação. Visando contribuir para um melhor conhecimento da espécie e da familia, bem como avaliar a provável influência dos fatores ambientais sobre a estrutura interna da planta é feito o estudo anatômico da espécie ocorrente em dois habitats distintos - o Horto Botânico do Museu Nacional (RJ) e a praia de Grumari (RJ). A espécie apresenta três diferentes formas foliares: peciolada, alada e séssil, posicionadas, respectivamente, na porção basal, mediana e apical do corpo da planta; anatomicamente, os exemplares dos dois ambientes exibem na lâmina foliar dos três tipos de folhas, um mesofilo dorsiventral, uma epiderme simples, com diferentes graus de sinuosidade das paredes celulares, estômatos predominantemente anisocíticos, tricomas 
simples, pluricelulares e longos, bordo com espessamento e silicificação; na região distal no ramo é visualizada uma endoderme com estrias de caspary, externamente aos canais secretores.

Palavras-chave: Emilia sonchifolia, anatomia vegetal, Asteraceae Agência Financiadora: CAPES

TÍTULO: Estudos morfológicos e taxinômicos do gênero Temnadenia Miers (Apocynaceae)

AUTOR: Luciene Bernardo Santos

DATA: $\quad 09 /$ outubro/1996

LOCAL: Universidade Federal do Rio de Janeiro, Museu Nacional, Departamento de Botânica, Rio de Janeiro, RJ

NÍVEL: Mestrado

BANCA EXAMINADORA: Margarete Emmerich- UFRJ (orientador)

Jorge Fontella Pereira - JBRJ

Luiza Sumiko Kinoshita - UNICAMP

Lúcia D'Ávila F. de Carvalho - JBRJ

RESUMO-O presente trabalho apresenta o estudo morfológico e taxonômico das espécies do gênero Temnadenia Miers, constantes de plantas escandentes encontradas na América do Sul. No Brasil ocorrem três espécies principalmente em cerrado e restinga. Na região sudeste e nordeste foi registrada $T$. violacea (Vell) Miers; nas regiões nordeste, sudeste e sul ( $T$. stellaris (Lindl.) Miers e na região norte, $T$. ornata (Hoehne) Woodson). T. stenantha Woodson ocorre no noroeste da Colômbia. O estudo engloba a morfologia externa, o padrão de anatomia foliar, a caracterização dos grãos de pólen e a análise da superficie da testa das sementes através da microscopia eletrônica. Os resultados obtidos diferenciaram as espécies pelas flores, relação altura do nectário e ovário, pilosidade das anteras, grãos de pólen e ornamentação da testa das sementes. O padrão de anatomia foliar confirmou as características já mencionadas para outros representantes da familia Apocynaceae Juss. e mostrou diferenças entre as espécies no parênquina paliçadico do mesofilo, de nervura mediana e nas formas celulares da parênquima lacunoso. Cada taxon estudado está documentado pelas fotografias, dados na fenologia, ilustrações e mapas da distribuição geográfica.

Palavras-chave: Apocynaceae, taxonomia Agência Financiadora: CAPES

TÍTULO: Estudo taxonômico de Humiraceae no Estado do Rio de Janeiro, Brasil

AUTOR: Luiz Carlos da Silva Giordano

DATA: $\quad 02 /$ agosto/1996

LOCAL: Universidade Federal do Rio de Janeiro, Museu Nacional, Departamento de Botânica, Rio de Janeiro, RJ

NÍVEL: Mestrado

BANCA EXAMINADORA: Graziela Maciel Barroso - JBRJ (orientador)

Luiz Emygdio de Mello Filho - UFRJ

Jorge Fontella Pereira - JBRJ

Lúcia D'Ávila Freire de Carvalho - JBRJ

RESUMO - Humiriaceae para o Estado do Rio de Janeiro registra a ocorrência de nove táxons. O tratamento taxonômico destes compreende chaves analiticas para a identificação dos mesmos, descrições, ilustrações relevantes das folhas, flores e frutos, fotografias de algumas exsicatas, inclusive dos tipos, dos habitats e hábitos, além de comentários taxonômicos importantes e mapas das áreas de ocorrência. É proposto e comentado um novo sinônimo e ainda sugerida a hipótese de um híbrido natural entre duas variedades. Algumas novas tipificações são realizadas. Dados anatômicos, ecológicos e fenológicos são apresentados, assim como informações sobre distribuição geográfica e nomes populares, além de 
comentários sobre os valores econômicos e medicinais atribuidos às entidades. Comenta-se ainda o procedimento de insetos ao visitarem as flores de dois táxons e a germinação de um deles. Faz-se menção da distribuição de cada táxon atualmente no Estado do Rio de Janeiro em comparação à antiga, relacionando-a com a conservação dos ecossistemas de floresta pluvial atlântica e restinga.

Palavras-chave: taxonomia, Humiriaceae

Agência financiadora: $\mathrm{CNPq}$

TíTULO: Composição florística e estrutura do estrato arbóreo de uma floresta situada na zona da mata mineira, Municipio de Lima Duarte, MG

AUTOR: Vinicius Campos de Almeida

DATA: $\quad 04 /$ setembro/1996

LOCAL: Universidade Federal do Rio de Janeiro, Museu Nacional, Departamento de Botânica, Rio de Janeiro, RJ

NÍVEL: Mestrado

BANCA EXAMINADORA: Dorothy Sue Dunn de Araujo - FEEMA (orientador)

Margarete Emmerich- UFRJ

Ruy José Válka Alves - UFRJ

Fábio Rubio Scarano - UFRJ

RESUMO-Utilizando-se do método de parcelas para o estudo da composição florística e estrutura do componente arbóreo de um trecho de Mata Atlântica, amostrou-se um ha de mata, no qual, considerando todos os indivíduos com diâmetro igual ou superior a $5 \mathrm{~cm}$, foram encontrados 1932 indivíduos pertencentes a 225 espécies, 120 gêneros e 53 familias. As espécies mais importantes (com maior IVI) foram: Copaifera trapezifolia, Heisteria silvianii, Trichilia aff. casaretti, Chorisia speciosa, Aparisthmium cordatum, Euterpe edulis, Virola oleifera, Tapirira guianensis, Ocotea aciphylla, $O$. corymbosa e $O$. variabilis. As três famílias com maior número de indivíduos foram Myrtaceae (com 212), Euphorbiaceae (158) e Lauraceae (136). As três com maior número de espécies foram Myrtaceae (com 23), Lauraceae (18) e Rubiaceae (16). As familias com maior IVI foram Euphorbiaceae, Lauraceae, Myrtaceae, Meliaceae, Caesalpiniaceae, Annonaceae, Rubiaceae, Bombacaceae, Olacaceae e Mimosaceae. O indice de diversidade de Shannon ( $\mathrm{H}^{\prime}$ ) foi de $4,67 \mathrm{e}$ a equabilidade (J), foi de 0,86 . A mata apresentou um dossel descontinuo com indivíduos de até $30 \mathrm{~m}$, existindo acima destes, indivíduos emergentes com até $45 \mathrm{~m}$.

Palavras-chave: Mata Atlântica, fitossociologia, floristica

Agência financiadora: CAPES

TíTULO: Composição floristica e fitossociológica de um trecho de Mata Atlântica na Reserva Biológica do Tinguá, Nova Iguaçu, Rio de Janeiro

AUTOR: Hermanny Carneiro Rodrigues

DATA: $\quad 30 /$ agosto/1996

LOCAL: Universidade Federal do Rio de Janeiro, Museu Nacional, Departamento de Botânica, Rio de Janeiro, RJ

NÍVEL: Mestrado

BANCA EXAMINADORA: Ariane Luna Peixoto - UFRRJ (orientador)

Luiz Emygdio de Mello Filho - UFRJ

Dorothy Sue D. de Araújo - FEEMA

Fábio Rubio Scarano - UFRJ

RESUMO - Levantamento floristico e fitossociológico de um trecho de Mata Atlântica, na Reserva Biológica do Tinguá, Nova Iguaçu, Rio de Janeiro. A região abrange cerca de 26000 ha, ilhada pelo crescimento dos municípios de Nova Iguaçu, Duque de Caxias, Miguel Pereira e Petropólis. O clima e quente e úmido é do tipo am, da classificação de Köppen. A textura do solo é franco-argilo-Arenosa. 
As análises químicas indicaram um solo de tendência álica, com baixos teores de $\mathrm{P}, \mathrm{K}, \mathrm{CA}$ e Mg e valores altos para $\mathrm{Al}$. O pH em água variou de 4,2 a 4,5. Os valores de carbono orgânico foram relativamente altos. A amostragem florístico-fitossociológica foi efetuada através do método dos quadrantes, sendo alocados 200 pontos. O critério de inclusão foi de indivíduos com diâmetro à altura do peito iguais ou superiores a 2,5cm. Dos 800 indivíduos amostrados, 19 encontravam-se mortos ainda de pé e os 781 vivos, distribuídos em 51 familias, 73 gêneros e 189 espécies. O índice de Shannon foi de 4,36. As familias de maior IVI foram Rubiaceae (33), Palmae (28,47), Lauraceae $(25,82)$, Nyctaginaceae $(24,95)$, Euphorbiaceae $(19,51)$, Piperaceae $(18,20)$, Myrtaceae $(17,30)$, Leguminosae $(16,23)$ e Meliaceae. Estas nove familias, juntamente com o grupo de mortas, totalizam $69,5 \%$ do IVI total.

Palavras-chave: Mata Atlântica, fitossociologia, florística

Agência financiadora: CNPQ

TÍTULO: Phyllanthus L. (Euphorbiaceae) espécies e subespécies ruderais ocorrentes no Municipio do Rio de Janeiro, Rio de Janeiro, Brasil

AUTOR: Denise Lelis Campos

DATA: $\quad 04 /$ novembro/1996

LOCAL: Universidade Federal do Rio de Janeiro, Museu Nacional, Departamento de Botânica, Rio de Janeiro, RJ

NÍVEL: Mestrado

BANCA EXAMINADORA: Margarete Emmerich - UFRJ (orientador)

Ruy José V. Alves - UFRJ

Jorge Pedro P. Carauta - FEEMA

Arline Souza de Oliveira - UFRJ

RESUMO - Nesta dissertação realizamos o levantamento das espécies e subespécies de Phyllanthus que ocorrem no Municipio do Rio de Janeiro. Phyllanthus amarus schum., Phyllanthus tenellus Roxb., Phyllanthus urinaria L. subsp. urinaria e Phyllanthus niruri subsp. lathyroides (HBK) Webster, conhecidas na medicina popular como quebra-pedra ou erva-pombinha, são estudadas em nivel morfológico e palinológico. Ultimamente elas vem sendo mundialmente pesquisadas por serem diuréticas e possuírem efeitos no tratamento da hepatite $\mathrm{B}$, diabetes e outros. O tipo de ramificação, a forma das folhas, catáfilos e estipulas, as estruturas presentes no androceu e gineceu, assim como os frutos e sementes foram usados para a delimitação dos táxons. O estudo palinológico também foi utilizado como subsídio à sistemática das respectivas espécies. A localização destes táxons em nosso município, assim como o estudo taxinômico realizado, tem como objetivo fornecer dados para futuras pesquisas botânicas, farmacológicas e fitoquímicas.

Palavras-chave: Phyllanthus, taxonomia, Euphorbiaceae

Agência financiadora: CAPES

TÍTULO: Anatomia dos nectários florais de Erythrina velutina Willd., Caesalpinia echinata Lam. e Tecoma stans (L.) H.B.K.

AUTOR: Áurea Kobayashi F. Peçanha

DATA: $\quad 05 /$ dezembro/1996

LOCAL: Universidade Federal do Rio de Janeiro, Museu Nacional, Departamento de Botânica,

Rio de Janeiro, RJ

NÍVEL: Mestrado

BANCA EXAMINADORA: Léa de Jesus Neves - UFRJ (orientador)

Luiz Emygdio de Mello Filho - UFRJ

Rosa Fuks - JBRJ

Heloisa Alves de L. Carvalho - UFRJ 
RESUMO-É feito o estudo da anatomia floral de Erythrina velutina Willd., Caesalpinia echinata Lam. e Tecoma stans (L) H.B.K. objetivando o reconhecimento, ao nivel morfológico e histológico dos nectários dessas espécies. Os testes histoquímicos realizados identificaram os principais compostos ergásticos contidos nos tecidos nectariferos. O estudo revelou a ocorrência de nectários estruturais e não estruturais posicionados em diferentes regiões da estrutura floral em Erythrina velutina e Caesalpinia echinata. Foram identificados nectários talâmicos sendo estrutural em Erythrina velutina e mesofilário em Caesalpinia echinata. Nesta espécie foi reconhecido também nectário carpelar representado pelos tecidos que margeiam o lóculo; em Tecoma stans os nectários são do tipo estrutural: estes correspondem às escamas posicionadas nas sépalas e tricomas no tubo da corola o nectário estrutural corresponde a um disco hipoginico.

Palavras-chave: anatomia floral, Caesalpinia echinata Lam, nectários florais, Tecoma stans (L.) H.B.K. Agência financiadora: CAPES

TÍTULO: Hydrocotyle emarginata Vell. Considerações morfológicas e anatômicas

AUTOR: Rose Mary B. Pinto

DATA: $\quad$ 18/dezembro/1996

LOCAL: Universidade Federal do Rio de Janeiro, Museu Nacional, Departamento de Botânica, Rio de Janeiro, RJ

NIVEL: Mestrado

BANCA EXAMINADORA: Cecília Gonçalves Costa - JBRJ (orientador)

Léa de Jesus Neves - UFRJ

Lygia Dolores R. de S. Fernandes - UFRJ

Maria da Conceição Valente - JBRJ

RESUMO-No trabalho em pauta, são abordados aspectos morfológicos e anatômicos de exemplares de Hidrocotyle emarginata Vell. (Umbelliferae), crescendo em ambiente terrestre e aquático. Os individuos procedentes de ambiente terrestre se desenvolvem em local umbroso, enquanto aqueles que crescem em meio aquático estão expostos à ação direta dos raios solares. A análise anatômica do eixo vegetativo desses exemplares evidencia alguns aspectos comuns: a presença de uma endoderme típica em todos os órgãos estudados, canais secretores no caule, peciolo e lâmina foliar, bem como hidatódios na face abaxial da lâmina foliar. Entre os caracteres distintivos, citam-se: maior superfície foliar e menor espessura do mesofilo, com paliçádico menos conspicuo e maior proporção de amido no material de ambiente terrestre. Jả no material de ambiente aquático, observam-se espaços intercelulares maiores e mais regulares e estômatos situados ao mesmo nível ou levemente acima das demais células epidérmicas, sendo as câmaras subestomáticas mais evidentes. A plasticidade constatada é considerada como produto da interação entre os fatores hereditários e a ação do meio no desenvolvimento dos indivíduos analisados.

Palavras-chave: anatomia, eixo vegetativo, Hydrocotyle emarginata

Agência financiadora: CAPES

TíTULO: Revisão taxonômica do gênero Hindsia Bentham (Rubiaceae, Hedyotideae)

AUTOR: Fernando Régis Di Maio

DATA: $\quad 14 /$ janeiro/1997

LOCAL: Universidade Federal do Rio de Janeiro, Museu Nacional, Departamento de Botânica, Rio de Janeiro, RJ

NIVEL: Mestrado

BANCA EXAMINADORA: Ariane Luna Peixoto - UFRRJ (orientador)

Jorge Fontella Pereira - JBRJ

Graziela Maciel Barroso - JBRJ

Ruy José V. Alves - UFRJ 
RESUMO- São apresentados dados morfológicos e taxonômicos das espécies do gênero Hindsia Bentham, que faz parte da tribo Hedyotideae, subfamilia Rubiodeae, familia Rubiaceae. O gênero é exclusivamente brasileiro, sendo composto por 11 espécies arbustivas que habitam campos de altitude, campos rupestres e vegetações de transição para estes ecossistemas. O estudo taxonômico baseou-se na análise de exsicatas de coleções de diversos herbários nacionais e estrangeiros, tipos e fotografias de tipos, bem como exemplares observados em campo. Incluem-se diversas questões de taxonomia, ecologia e fitogeografia. É proposta a sinonimização de uma espécie e a descrição de cinco espécies novas para a ciência.

Palavras-chave: taxonomia, Rubiaceae, Hindsia

Agência financiadora: $\mathrm{CNPq}$

TÍTULO: Biologia da reprodução de Swartzia apetala Raddi var. apetala (Leguminosae Caesalpinioideae)

AUTOR: Maria Cecília de C. Moço

DATA: $\quad 30 /$ janeiro/1997

LOCAL: Universidade Federal do Rio de Janeiro, Museu Nacional, Departamento de Botânica,

NIVEL: Mestrado

BANCA EXAMINADORA: Maria Célia B. Pinheiro - UFRJ (orientador)

Wilma Texeira Ormond - UFRJ

Vania Gonçalves L. Esteves - UFRJ

Heloísa Alves de L. Carvalho - UFRJ

RESUMO- Este estudo abrange a biologia floral, as atividades dos visitantes florais e o sistema de reprodução de Swartzia apetala Raddi var. apetala com o objetivo de compreender as causas do aborto de frutos e sementes. São feitas ainda considerações sobre o padrão fenológico da floração e frutificação. As flores são hermafroditas, zigomorfas e odoríferas. O androceu é dimorfo e atrativo. A floração segue o padrão anual assincrônico em nivel populacional e ocorre entre os meses de novembro e março. As flores são polinizadas através do método de vibração por abelhas solitárias e poliléticas, sendo as principais Xylocopa ordinaria e Centris flavifrons. A variedade é auto-incompativel. Dos frutos oriundos de polinizações xenogâmicas manuais $82.95 \%$ abortaram gradualmente até a oitava semana. A baixa eficiência da polinização teve influência no número de frutos formados. Diversas hipóteses referentes ao aborto seletivo de frutos e sementes, e a disponibilidade de recursos maternos são mencionadas.

Palavras-chave: biologia da reprodução, aborto de frutos, Leguminosae Agência financiadora: $\mathrm{CNPq}$

TíTULO: Aspectos etnobotânicos numa área rural de São João da Cristina, MG

AUTOR: Lidice Meyer P. Ribeiro

DATA: $\quad 02 /$ fevereiro/1997

LOCAL: Universidade Federal do Rio de Janeiro, Museu Nacional, Departamento de Botânica,

NÍVEL: $\quad$ Rio de Janto

BANCA EXAMINADORA: Margarete Emmerich - UFRJ (orientador)

Luiz Emygdio de Mello Filho - UFRJ

Margarida Maria Moura - USP

Nuno Ảlvares Pereira - UFRJ

RESUMO-O presente trabalho é o resultado do levantamento das espécies vegetais utilizadas por uma comunidade rural do sul de Minas Gerais. O bairro de São João da Cristina pertence ao Municipio de Maria da Fé, MG, sendo um povoado camponês e tendo como centro da vida social uma Igreja Protestante. 
Foram levantadas 107 espécies pertencentes a 42 famílias. Destas, observou-se a ocorrência de 100 plantas de uso medicinal, quatro forrageiras, três tóxicas, duas comestiveis e uma tintorial. São fornecidos para cada espécie o nome cientifico e vulgar, a descrição, a distribuição geográfica, o hábito, o habitat, a utilidade local, pesquisas farmacológicas e outras observações. Nas plantas medicinais procurou-se conservar a interpretação popular relativa à identificação e cura de uma dada doença, o modo de preparo e aplicação. Os dados estão organizados em tabelas e gráficos. Esta pesquisa revelou a riqueza do conhecimento etnobotânico camponês, tanto ao nivel da diversidade de recursos utilizados, como ao nível da utilização e manipulação destes recursos. Tal conhecimento pode fornecer subsidios para pesquisas mais aprofundadas em várias disciplinas e contribuir para o desenvolvimento de programas de saúde mais adaptados às comunidades rurais.

Palavras-chave: etnobotânica, plantas medicinais, Minas Gerais Agência financiadora: $\mathrm{CNPq}$

TÍTULO: Anatomia e histoquimica de Ficus aspera Forster Filius - Moraceae

AUTOR: Philomena Mariano Vieira

DATA: $\quad 04 / \operatorname{março/} 1997$

LOCAL: Universidade Federal do Rio de Janeiro, Museu Nacional, Departamento de Botânica,

Rio de Janeiro, RJ.

NÍVEL: Mestrado

BANCA EXAMINADORA: Léa de Jesus Neves - UFRJ (orientador)

Luiz Emygdio de Mello Filho - UFRJ

Maria da Conceição Valente - JBRJ

Jorge Pedro P. Carauta - FEEMA

RESUMO - Anatomia foliar de Ficus aspera F. sinonimia Ficus parcellii Veitch ex. Cogn. \& Marchal planta ornamental de folhagem variegada, cujo nome vulgar é figueira da polinésia ou mosaic fig em língua inglesa. O material estudado foi coletado no Rio de Janeiro, na Praça 15 de novembro. Anatomicamente a planta se caracteriza pela folha dorsiventral epigena hipostomática com textura cartácea tendo a superficie adaxial áspera e a superficie abaxial pubescente. O caráter híspido da folha é resultante da impregnação de sílica nas paredes das células epidérmicas, principalmente nos pêlos cistolíticos. Em F. aspera encontramos diferentes tipos de estruturas cistoliticas: pêlos, litocistos, cistólitos bem diferenciados e formações cistoliticas mais discretas ocorrendo em células epidérmicas não diferenciadas das demais. A presença de cistólitos em células do segundo estrato da epiderme adaxial (ventral) é ocorrência, até a presente data, só reconhecida em $F$. aspera. Esta espécie apresenta variação na estrutura da lâmina de acordo com o padrão variegado das folhas de sol, tendo sido possivel distingüir dois padrões estruturais básicos, dentre os quais uma gama de gradações pode ser observada. As áreas brancas apresentam menor espessura das paredes celulares na epiderme, menor número de estratos no mesofilo, parênquima desclorofilado com células mais espaçadas, tem sistema vascular e mecânico menos conspicuos e pouco lignificado.

Palavras-chave: Ficus aspera, Ficus parcellii, anatomia foliar, folhas variegadas Agência financiadora: CAPES

TíTULO: Taxonomia e morfologia de Ectocarpus breviarticulatus, Feldmannia indica, Feldmannia irregularis, Hincksia conifera e Hincksia mitchelliae (Ectocarpaceae, Phaeophyta) no Estado do Rio de Janeiro

AUTOR: Valéria Cassano

DATA: $\quad 02 /$ abril/1997

LOCAL: Universidade Federal do Rio de Janeiro, Museu Nacional, Departamento de Botânica, Rio de Janeiro, RJ

NÍVEL: Mestrado 
BANCA EXAMINADORA: Yocie Yoneshigue - Valentin - UFRJ (orientador)

Mariângela Menezes - UFRJ

Valéria Laneuville Teixeira - UFF

Renato Crespo Pereira - UFF

RESUMO - As espécies foram estudadas em seis estações de coleta ao longo do litoral do Estado do Rio de Janeiro, através de coletas periódicas, realizadas na zona entremarés até a franja do infralitoral, entre 1993 e 1996. Este trabalho teve por objetivo estudar a morfologia dos gêneros e suas espécies, suas variações morfológicas, e avaliar os critérios utilizados para a taxonomia dos gêneros. Foi realizado cultivo in vitro de espécies com problemas taxonômicos com o objetivo de auxiliar a identificação dos táxons e sua variabilidade morfológica. Foram estudados cinco táxons de categoria infragenérica, sendo dois pertencentes ao gênero Hincksia: $H$. conifera e H. mitchelliae, e dois ao gênero Feldmannia: F. irregularis e $F$. indica. A espécie Ectocarpus breviarticulatus foi mantida sob o seu nome mais antigo pela evidenciação de plastos em forma de fita, em cultivo. A plasticidade morfológica dos gêneros, tanto nas plantas de campo quanto nas de cultivo, foi confirmada

Palavras-chave: algas marinhas, cultivo, Ectocarpaceae

Agência financiadora: $\mathrm{CNPq}$

\section{Universidade Estadual Paulista - UNESP - Botucatu}

TíTULO: Organogênese em Eucalyptus

AUTOR: Clóvis José Fernandes de Oliveira Júnior

DATA: $\quad 03 /$ março/1997

LOCAL: Universidade Estadual Paulista - Instituto de Biociências - Campus de Botucatu-SP

NIVEL: Mestrado

BANCA EXAMINADORA： Oswaldo Galvão Brasil - IB, UNESP, Botucatu (orientador)

João Domingos Rodrigues -IB, UNESP, Botucatu-SP

Luis Carlos Basso - ESALQ, USP

RESUMO - O objetivo deste trabalho foi estudar a Bioquimica e Fisiologia de E. grandis $x$ E.. urophila, colaborando para seu entendimento. Foram induzidos calos em três tipos de meio de cultura, a partir de folhas, hipocótilos, cotilédones e raizes retirados de plântulas cultivadas in vitro. Os calos formados foram repicados em dois tipos de meiọ para regeneração, totalizando seis combinações de meio de indução e meio de regeneração para cada um dos quatro tipos de explante. No fim do periodo de formação de calos (30 dias) e ao fim do cultivo em meio de regeneração (60 dias) foram retiradas amostras para análise de percentagem da matéria seca e para as análises bioquimicas (teor de açúcares redutores, teor de proteina solúvel e atividade de peroxidase). A utilização de hipocótilo como explante foi mais favorável à regeneração de gemas para diferenciação de raizes; o melhor tipo de explante foi a raiz. Os tratamentes utilizados neste experimento afetaram os teores de açúcares redutores, de proteína solúvel e a atividade da peroxidase. Calos que apresentaram organogênese mostraram maiores níveis de açúcares redutores, assim como calos que formaram gemas. Aos 30 dias apresentaram maiores teores de proteina solúvel. A atividade da peroxidase foi maior em calos que diferenciaram gemas e raizes aos 60 dias.

Palavras-chave: organogênese, Eucalyptus, peroxidase, proteína e açúcares redutores Agência Financiadora: $\mathrm{CNPq}$

TÍTULO: Efeito do virus do enrolamento da folha da batata sobre os teores de poliaminas, proteínas, açucares e atividade da peroxidase em cultivares de batata (Solanum tuberosum L.) com diferentes niveis de resistência
AUTOR:
Jurema Schons
DATA:
11/abril/1997 